CAUSATION

\title{
A decrease in care was associated with suicide in people with mental illness
}

\author{
Appleby L, Dennehy JA, Thomas CS, et al. Aftercare and clinical characteristics of people with mental illness who commit suicide: a \\ case-control study. Lancet 1999 A pr 24;353:1397-1400.
}

\section{Question}

In people with severe mental illness who commit suicide, what is the relation of suicide to aspects of mental health care, clinical features, and historical variables?

\section{Design}

Case control study.

\section{Setting}

Greater Manchester area, UK.

\section{Participants}

Cases were 149 people (median age 38 y) who committed suicide in Greater Manchester within 5 years of discharge from inpatient psychiatric care. Controls were 149 discharged psychiatric inpatients matched to cases by age (within 5 y), sex, clinical diagnosis, and date of admission (within $6 \mathrm{mo}$ ).

\section{Assessment of risk factors}

Historical variables, clinical features since final inpatient admission, and details about care after inpatient discharge (aftercare). Care variables included components of care at the time of suicide, decreases in care (decrease in frequency of follow up and drug dosage, transfer to a setting with less observation, and discharge from follow up), and corresponding increases in care. Predictable risk (risk of suicide perceived by a clinician at the last contact before suicide) was rated independently by 2 psychiatrists blinded to case or control status.

Main outcome measure

Risk of suicide.

\begin{abstract}
Main results
The historical and clinical variables that were most strongly associated with suicide were index admission as the first psychiatric admission, history of self harm, and suicidal thoughts during aftercare. These risk factors and depression in the 3 months before suicide were also associated with each other, and therefore adjustment was made (table). Predictable risk rated as moderate or high was not associated with suicide after adjusting for main risk factors. Patients who committed suicide were more likely to have had a decrease in care at their final appointment (table).
\end{abstract}

\section{Conclusion}

In people with severe mental illness who commit suicide, certain clinical features and historical variables were associated with suicide, including a decrease in care at their final appointment before death.

Risk factors associated with suicide in mental illness

\begin{tabular}{ll}
\hline Risk factors & Odds ratio $(95 \% \text { CI })^{*}$ \\
\hline $\begin{array}{l}\text { Index admission as first psychiatric } \\
\quad \text { admission }\end{array}$ & $2.00(1.12$ to 3.60$)$ \\
History of self harm & $3.05(1.65$ to 5.65$)$ \\
Suicidal thoughts during aftercare & $1.89(1.02$ to 3.51$)$ \\
Decrease in care & $3.73(1.82$ to 7.65$) \dagger$ \\
\hline
\end{tabular}

*Adjustment for history of self harm, suicidal thoughts during aftercare, index admission as first psychiatric admission, and depression in 3 months before suicide. †Additional adjustment for predictable risk of suicide.

Source of funding: North West NHS Trust Executive.

For correspondence: Professor L Appleby, School of Psychiatry and Behavioural Sciences, University Hospital of South Manchester, West Didsbury, Manchester M20 8LR, UK. Fax +44 (0)161 4459263.

A modified version of this abstract appears in Evidence-Based Mental Health

\section{Commentary}

Identifying people at high risk of suicide and reducing the numbers at risk are high priorities for mental health professionals in many countries. This case control study by Appleby et al permits examination of the relation between mental health service provision and suicide.

The sample of people who committed suicide and their matched controls were drawn from discharged psychiatric inpatients in Greater Manchester, UK. In a 30 month period, 708 overall confirmed or probable deaths occurred from suicide; of these, 149 suicides occurred in people who had had psychiatric hospital admissions within the previous 5 years. The authors used a model that linked risk of suicide to specific clinical predictive factors. The predictable risk of suicide, defined as risk as it would have been per- ceived by a clinician at the last contact before suicide, was independently assessed by review of clinical notes by 2 psychiatrists who were unaware of which patient was a case and which was a control. The authors acknowledged the limitation of using clinical notes but also took steps to address factors that might have confounded their findings.

The results are relevant to psychiatric and mental health nurses who work in clinics, outpatient or day care programmes in the community, and hospitals. The age range of the patients was 16-93 years (median 38 y). Because those at highest risk for suicide tend to be young men and elderly men, these findings are also important to nurses working with adolescent and elderly populations. The finding critical to clinicians is that there was more likely to have been a decrease in care at the time of the last appointment for those who committed suicide. This has important implications for practice.

Study findings suggest that clinicians maintain the intensity of aftercare for up to 1 year after hospital discharge, even beyond perceived clinical recovery. In light of our current inability to identify those at highest risk of suicide, this study also has implications for administrators to support aftercare programmes. Researchers need to continue to identify those aftercare factors that may be part of the multifaceted approach needed to make a difference.

Diane Buchanan, RN, DNS

Clinical Nurse Specialist, Nurse Researcher Baycrest Centre for Geriatric Care Toronto, Ontario, Canada 